\title{
MODELO MATEMÁTICO DE LETALIDAD SOBRE BACILLUS ARTHOPHAEUS EN PROCESOS DE ESTERILIZACIÓN DE DISPOSITIVOS MÉDICOS CON ÓXIDO DE ETILENO
}

\author{
$\checkmark$ NATALIA JARAMILlO-RUIZ ${ }^{1}$ \\ PEDRonel ARAQUe-Marín ${ }^{2}$ \\ YESID MONTOYA-GÓEZ ${ }^{3}$
}

\section{RESUMEN}

La esterilización de dispositivos médicos con óxido de etileno tiene como objetivo eliminar toda forma de vida que pueda tener contacto con los tejidos corporales, destruyendo la contaminación microbiana que podría dar lugar a la transmisión de enfermedades. La esterilización con óxido de etileno se realiza a bajas temperaturas y se aplica a los dispositivos médicos que no pueden ser esterilizados con vapor de agua. A demás de ser un proceso costoso, la esterilización con óxido de etileno presenta algunas desventajas al ser implementada con ciclos de tiempos prolongados, convirtiéndose en un factor de riesgo para los pacientes y el personal médico. El presente estudio propone un modelo matemático para medir y predecir la tasa de letalidad en procesos de esterilización de dispositivos médicos con OE en función de la concentración de óxido de etileno, humedad relativa, presión, temperatura de trabajo y el tiempo de exposición del dispositivo con óxido de etileno. El modelo matemático propuesto describe un comportamiento cinético de primer orden, para un numero de esporas de Bacillus arthophaeus de 3.5 $\times 10^{6}$, una concentración de óxido de etileno de $700 \mathrm{ppm}$, una temperatura ambiente de $23{ }^{\circ} \mathrm{C}$, una temperatura de trabajo de $60{ }^{\circ} \mathrm{C}$, una humedad relativa del $60 \% \mathrm{y}$ una presión de trabajo 0.490 atm.

PALABRAS CLAVE: modelo matemático, esterilización, óxido de etileno, tasa de letalidad, Bacillus arthophaeus, dispositivo médico.

1 Ingeniera Biomédica, Universidad EIA. Candidata a Magister en Ingeniera Biomédica, Universidad EIA. Investigadora. Grupo de Investigación en Ingeniería Biomédica EIA-CES (GIBEC), Universidad EIA, Envigado, Colombia.

2 Químico, Universidad de Antioquia. Magister en Ciencias Químicas, Universidad de Antioquia. Candidato a Doctor en Ciencias Químicas, Universidad de Antioquia. Director Grupo de Investigación en Ciencias Básicas y Aplicadas, Universidad EIA. Profesor de Planta, Universidad EIA, Envigado, Colombia.

3 Ingeniero Civil, Universidad Nacional de Colombia - Sede Medellín. Magister en Ciencias de los Materiales Universidad Nacional de Colombia - Sede Medellín. Investigador. Grupo de Investigación en Ingeniería Biomédica EIA-CES (GIBEC), Universidad EIA. Profesor de Planta, Universidad EIA, Envigado, Colombia.

Autor de correspondencia: Jaramillo, N. (Natalia): Universidad EIA: Sede de Las Palmas: Km $2+200$ Vía al Aeropuerto José María Córdova. Envigado, Colombia. Código Postal: 055428. Tel.: (574) 30535 90, Ext. 1443. Correo electrónico: nataliajaramilloruiz@gmail.com
Historia del artículo:

Artículo recibido: 07-IV-2016 / Aprobado: 07-III-2018

Disponible online: 14 de marzo de 2018

Discusión abierta hasta abril de 2019 


\section{MATHEMATICAL MODEL OF LETHALITY AGAINST BACILLUS ATROPHAEUS IN STERILIZATION PROCESSES OF MEDICAL DEVICES WITH ETHYLENE OXIDE}

\section{ABSTRACT}

Sterilization of medical devices with ethylene oxide aims to eliminate all forms of life that may have contact with body tissues, destroying microbial contamination that could lead to disease transmission. Ethylene oxide sterilization is performed at low temperatures and it's applied to medical devices that cannot be steam sterilized. Besides being an expensive process, Ethylene oxide sterilization has some disadvantages when implemented with prolonged time cycles, becoming a risk factor for patients and medical staff. This study proposes a mathematical model to measure and predict the lethal rate in the process of ethylene oxide sterilization of medical devices based on its concentration, relative humidity, pressure, working temperature and exposure time of the device to the ethylene oxide. The proposed mathematical model describes a first-order kinetic behavior, for a number of spores of Bacillus arthophaeus of 3.5x10 ${ }^{6}$, a concentration of ethylene oxide of $700 \mathrm{ppm}$, an ambient temperature of $23^{\circ} \mathrm{C}$, a working temperature of $60{ }^{\circ} \mathrm{C}$, a relative humidity of $60 \%$ and a pressure $0.490 \mathrm{~atm}$.

KEYWORDS: mathematical model, sterilization, ethylene oxide, lethal rate, Bacillus arthophaeus, medical device.

\section{MODELO MATEMÁTICO DE LETALIDADE CONTRA BACILLUS ATROPHAEUS EM PROCESSOS DE ESTERILIZAÇÃO DE DISPOSITIVOS MÉDICOS COM ÓXIDO DE ETILENO}

\section{RESUMO}

Esterilização de dispositivos médicos com óxido de etileno visa eliminar todas as formas de vida que possa ter contacto com os tecidos do corpo, destruindo a contaminação microbiana que poderia levar a transmissão da doença. esterilização por óxido de etileno é realizada a baixas temperaturas e é aplicado a dispositivos médicos que não podem ser esterilizados por vapor. Além de ser um processo caro, esterilização por óxido de etileno tem algumas desvantagens quando implementado com ciclos de tempo prolongados, tornando-se um fator de risco para os pacientes e pessoal médico. Este estudo propõe um modelo matemático para medir e prever a taxa letal no processo de esterilização por óxido de etileno de dispositivos médicos com base na sua concentração, a humidade relativa, a pressão, a temperatura de trabalho e tempo de exposição do dispositivo ao óxido de etileno. 0 modelo matemático proposto descreve um comportamento cinético de primeira ordem, para um número de esporos de Bacillus arthophaeus de $3.5 \times 10^{6}$, uma concentração de óxido de etileno de $700 \mathrm{ppm}$, com uma temperatura ambiente de $23^{\circ} \mathrm{C}$, uma temperatura de funcionamento de $60^{\circ} \mathrm{C}$, uma humidade relativa de $60 \%$ e um 0.490 atm pressão.

PALAVRAS-CHAVE: modelo matemático, esterilização, óxido de etileno, a taxa letal, Bacillus arthophaeus, dispositivos médicos. 


\section{INTRODUCCIÓN}

La esterilización en dispositivos médicos tiene como objetivo eliminar toda forma de vida que pueda tener contacto con los tejidos corporales. Estos dispositivos deben ser estériles cuando se usan, ya que cualquier contaminación microbiana podría dar lugar a la transmisión de enfermedades (Forero et al., 1997), por lo anterior los dispositivos son sometidos a esterilización utilizando vapor de agua, ya que, al no ser toxico, cuenta con el mayor margen de seguridad, economía, esporicida y se calienta rápidamente y penetra en los tejidos del dispositivo. Sin embargo, otros elementos requieren el uso de sustancias químicas para la esterilización (Rutala y Weber, 1999). Se requiere la esterilización de baja temperatura para dispositivos sensibles a la humedad y altas temperaturas. El uso de óxido de etileno (OE) es un proceso de esterilización a baja temperatura utilizado para el reuso de dispositivos médicos que no pueden ser esterilizados con vapor, el OE es un gas incoloro, inflamable y explosivo que inactiva desde virus grandes con envoltura lipídica hasta esporas bacterianas, debido al remplazo de los átomos de hidrogeno con un grupo alquilo en las proteínas, ADN, ARN y en microorganismos mediante la unión a los sulfhidrilo e hidroxilo, amino y grupos carboxilo que dentro de las células impiden el metabolismo celular normal y la capacidad de reproducción, aunque la exposición al OE no causa cambios estructurales al dispositivo. La eficacia de la esterilización por $\mathrm{OE}$, junto con la flexibilidad del proceso, representa algunas de las ventajas de este método en la esterilización de dispositivos médicos, ya que los parámetros del ciclo de $\mathrm{OE}$ se pueden ajustar para preservar su integridad (Mendes, Brandão y Silva, 2007).

Las principales desventajas asociadas con OE son el tiempo de uso en ciclos largos, su alto costo y sus riesgos potenciales para los pacientes y el personal, ya que es tóxico, carcinógeno e inflamable (Rutala, Gergen y Weber, 1998). Para la esterilización con $\mathrm{OE}$ se necesitan equipos que garanticen parámetros de temperatura, humedad, tiempo de exposición, presión y concentración del agente, estos equipos de esterilización están compuestos de cinco ciclos; preacondicionamiento y humidificación, introducción del gas, exposición del OE, evaluación y aireación (Acosta-Gnass y Stempliuk, 2008).

La eficiencia de estos equipos depende de la programación de estos parámetros, ya que la programación inadecuada del ciclo podría llevar a una baja letalidad de microorganismos, al aumento de los costos y del tiempo por ciclos extremadamente largos y con uso excesivo de gas, además de la generación de trazas de OE que podrían causar daños en pacientes y operadores de los equipos de esterilización. Tanto Mendes et al. (2007) como Agalloco y Carleton (2007) describen diferentes modelos matemáticos que permiten validar y mejorar el proceso de esterilización con OE. Mendes et al. (2007) desarrollaron un modelo en función de los parámetros principales con funciones no específicas de concentración $C(t)$ y temperatura $T(t)$, por otra parte solo es tenido en cuenta el Valor-D de referencia y la humedad no es considerada como variable.

En cuanto a Agalloco y Carleton (2007) no sintetizan la letalidad en una formula, sino que describe cada parámetro independientemente (presión, concentración, humedad, temperatura) y no logran relacionar la humedad con la letalidad de los microorganismos. Se considera entonces que es necesario un modelo que integre todas las variables que influyen en la muerte microbiana, con el principal objetivo de arrojar resultados aproximados que permitan validar procesos y describir ciclos eficaces. El presente estudio propone un modelo matemático para medir y predecir la tasa de letalidad en procesos de esterilización de dispositivos médicos con $\mathrm{OE}$ en función de la concentración de $\mathrm{OE}$, humedad relativa, presión, temperatura de trabajo y el tiempo de exposición del dispositivo con $\mathrm{OE}$. 


\section{DESCRIPCIÓN DEL PROCESO}

\section{Deducción de la concentración de OE}

La cámara se simuló con $\mathrm{OE}_{(g)} \mathrm{y} \mathrm{CO}_{2(g)}$ como gas diluyente, proponiendo un comportamiento ideal de OE y la mezcla $\left[\mathrm{OE}_{(\mathrm{g})} ; \mathrm{CO}_{2(\mathrm{~g})}\right]$, para esto se utilizó la siguiente ecuación de estado para un gas ideal:

$$
P V=n R T
$$

Donde $P$ es la presión del gas en Bar; $V$ es el volumen que ocupa el gas en Litros; $n$ las moles del gas; $R$ la constante universal de los gases ideales cuyo valor para este trabajo es $0.08314 \mathrm{Bar} . \mathrm{L} / \mathrm{mol} . \mathrm{K}$ y $T$ es la temperatura en Kelvin.

\section{Deducción de la presión en función de la concentración de $\mathrm{OE}$}

La presión es un método indirecto que permite controlar la concentración, es decir cuando la cámara es presurizada se asume que el interior contiene el porcentaje dado de $\mathrm{OE}$ relativo al cambio en la presión (Agalloco y Carleton, 2007). Dada la dificultad de modelar la concentración dentro de la cámara, se acude a la proporcionalidad de $\mathrm{P}$ y $\mathrm{C}$ de los gases ideales y de esta forma es posible la manipulación de la presión para ajustar le concentración de OE deseada.

\section{Deducción de la presión en función de humedad relativa}

Para la esterilización con OE se pre-acondiciona la cámara de esterilización garantizando temperatura y humedad en el sistema por inyección de vapor de agua para proporcionar un ambiente apto para la muerte microbiana, por ende, es necesario determinar la presión real contenida en el ambiente y en la cámara de esterilización en términos de la humedad relativa (\%HR) (Kaiser et al., 2002), como se indica a continuación:

$$
\% H R=\frac{P_{v}}{P_{s}} \times 100 \%
$$

Donde $P_{v}$ es la presión contenida en el aire a una temperatura específica y $P_{s}$ es la presión de vapor de agua o también llamada presión de saturación.

\section{Deducción del Tiempo letal 90 (Valor} D) en función de la concentración de $O E$

El Valor D representa el tiempo en minutos requerido para reducir una población microbiana al $90 \%$, este valor disminuye a medida que la temperatura y las concentraciones de $\mathrm{OE}_{(\mathrm{g})}$ se incrementan (ISO 11135-1).

Para encontrar el Valor-D en función de la concentración, se debe tener en cuenta el Tiempo letal de referencia (Valor- $\mathrm{D}_{\mathrm{Ref}}$ ), el cual está dado por la ficha técnica de los indicadores biológicos usados como Bacillus atropheus, a una concentración, un $\% H R$ y una temperatura de referencia $\left(\mathrm{T}_{\text {Ref }}\right.$ ) (Agalloco y Carleton, 2007).

El Valor-D es inversamente proporcional a la concentración de $\mathrm{OE}$, como se expresa a continuación:

$$
\text { Valor } D=\frac{k}{C_{O E}}
$$

Cambio de la tasa de letalidad microbiana (Z)

El cambio de la tasa de letalidad microbiana se denomina Z y depende de la temperatura. Se interpreta como el número de grados que la temperatura debe incrementar para que el Valor-D cambie por un factor de 10 (Mendes, Brandão y Silva, 2007). Entre los rangos de temperatura $\left(40-60^{\circ} \mathrm{C}\right)$ y de concentración (300-750 ppm) el cambio de la tasa de letalidad tiene un valor de $31^{\circ} \mathrm{C}$ (Agalloco y Carleton, 2007).

\section{Deducción de la tasa de letalidad}

La tasa letal (TL) se define como el tiempo equivalente de un proceso a una temperatura de referencia de $54{ }^{\circ} \mathrm{C}$ con respecto a temperatura de trabajo (Agalloco y Carleton, 2007; Mendes, Brandão y Silva, 2007; Mendes y Brandao, 2012). La Ecuación 4, representa la tasa de letalidad para Bacillus atropheus a $\mathrm{T}_{\text {Ref }}=54^{\circ} \mathrm{C} \mathrm{y} \mathrm{Z}=31^{\circ} \mathrm{C}$. 


$$
T L_{\text {Ref }}=A * 10\left(\frac{T_{\text {trabajo }}-54^{\circ} \mathrm{C}}{31^{\circ} \mathrm{C}}\right)
$$

Donde $A$ es una constante de valor $=1$ minuto.

Deducción del Tiempo letal 90 (Valor D) en función de la temperatura

El cambio de la tasa de letalidad microbiana con respecto a la temperatura se plantea en la Ecuación 5, para $\mathrm{T}_{\text {Ref }}=54{ }^{\circ} \mathrm{C} \mathrm{y} \mathrm{Z}=31^{\circ} \mathrm{C}$. (Russel, Hugo, y Avliffe, 2004; Agalloco y Carleton, 2007):

$$
\text { Valor } D_{\text {Ref }}=\text { Valor } D * 10\left(\frac{T_{\text {trabajo }}-54{ }^{\circ} \mathrm{C}}{31^{\circ} \mathrm{C}}\right)
$$

\section{Modelación del sistema}

El programa utilizado para la modelación de la letalidad fue Matlab R2013a (8.1.0.604).

\section{RESULTADOS}

\section{Concentración de óxido de etileno $\left(C_{O E}\right)$}

Teniendo en cuenta que la mezcla de gases dentro de la cámara se comporta como un gas ideal, y que los gases conservan la identidad debido a la absorción, adsorción y/o condensación, se estableció que la presión total de una mezcla de gases es la suma de las presiones parciales de los componentes de la mezcla (La ley de las presiones parciales de Dalton):

$$
P_{T}=P_{O E}+P_{C O_{2}}
$$

Expresando la presión de cada gas en términos de la concentración utilizando la Ecuación 1:

$$
P_{T}=\left(\frac{n}{v}\right)_{O E} R T+\left(\frac{n}{v}\right)_{C O_{2}} R T
$$

Donde $P_{O E}$ es la presión de $\mathrm{OE}_{(\mathrm{g})}$ y $P_{C O 2}$ es la presión del gas diluyente $\mathrm{CO}_{2(\mathrm{~g})}, R$ es la constante de los gases ideales $R=0.08314 \mathrm{Bar} . L / \mathrm{mol} . \mathrm{Ky}$ T es la temperatura en Kelvin.

Teniendo en cuenta que la masa molecular de $\mathrm{OE}_{(\mathrm{g})}$ es aproximadamente igual a la masa molecular del $\mathrm{CO}_{2(\mathrm{~g})}$ la Ecuación 7 se puede expresar como:

$$
P_{T}=\frac{R T}{44 \mathrm{~g} / \mathrm{mol}}\left(\frac{m}{v}\right)_{O E}+\frac{R T}{44 \mathrm{~g} / \mathrm{mol}}\left(\frac{m}{v}\right)_{\mathrm{CO}_{2}}
$$

Como $1 \mathrm{mg}$ son $1 \times 10^{-3} \mathrm{~g}$ y reemplazando en la

Ecuación 8, tenemos:

$$
P_{T}=\frac{R T}{44 \mathrm{~g} / \mathrm{mol}} * 1 \times 10^{-3}\left[\left(\frac{m}{v}\right)_{O E}+\left(\frac{m}{v}\right)_{\mathrm{CO}_{2}}\right]
$$

(Agalloco y Carleton, 2007) (Agalloco y Carleton, 2007)(Agalloco y Carleton, 2007).

$\mathrm{Si}$ describimos el contenido porcentual en masa de OE en la mezcla como:

$$
\% O E=\frac{m_{O E}}{m_{O E}+m_{C O_{2}}} * 100 \%
$$

(Agalloco y Carleton, 2007)(Agalloco y Carleton, 2007)(Agalloco y Carleton, 2007).

Teniendo en cuenta que el volumen de la mezcla es el volumen que ocupa cada gas tenemos:

$$
\% O E=\frac{\left(\frac{m}{v}\right)_{O E}}{\left(\frac{m}{v}\right)_{O E}+\left(\frac{m}{v}\right)_{C O_{2}}} \times 100 \%
$$

Modificando 11:

$$
\left(\frac{m}{v}\right)_{O E}+\left(\frac{m}{v}\right)_{C O_{2}}=\frac{\left(\frac{m}{v}\right)_{O E}}{\% O E} \times 100 \%
$$

Sustituyendo 12 en 9:

$$
P_{T}=\frac{R T}{44 \mathrm{~g} / \mathrm{mol}}\left(\frac{1 \times 10^{-3}}{\% O E}\right)\left(\frac{m}{v}\right)_{O E} \times 100 \%
$$

Simplificando 13

$$
P_{T}=\frac{1}{440 \mathrm{~g} / \mathrm{mol} * \% O E}(R T)\left(\frac{m}{v}\right)_{O E}
$$

De donde se obtiene el valor $K$ llamado la constante de la mezcla gaseosa:

$$
K=440 \mathrm{~g} / \mathrm{mol} * O E
$$


De esta forma se expresa la concentración de OE como:

$$
C_{O E}[p p m]=\frac{P_{T} K}{R T}=\left(\frac{m}{v}\right)_{O E}
$$

Dado que existen valores de \%OE entre 10\% y 90\% en los diferentes ciclos de esterilización (Agalloco y Carleton, 2007), para este modelo se desea trabajar con una mezcla de gases $90 \% \mathrm{OE}_{(\mathrm{g})}$ y $10 \%$ $\mathrm{CO}_{2(\mathrm{~g})}$, por lo tanto la constante $K$ adquiere un valor de $39600 \mathrm{~g} / \mathrm{mol}$.

\section{Presión de trabajo en función de la} concentración de $\mathrm{OE}$

De la Ecuación 16 se obtiene de la deducción del sistema de presiones que controlan la concentración y se describe de la siguiente forma:

$$
\begin{gathered}
P_{\text {Tamb }}=\frac{R * T_{a m b} * C_{O E}}{K} \\
P_{\text {Ttrabajo }}=\frac{R * T_{\text {trabajo }} * C_{O E}}{K}
\end{gathered}
$$

Donde $P_{\text {Tamb }}$ es la presión en Bar a la que se encuentra la cámara a temperatura ambiente $\left(T_{a m b}\right)$ y $P_{\text {Ttrabajo }}$ es la presión en Bar a la que se encuentra la cámara a la temperatura de la cámara $\left(T_{\text {trabajo }}\right)$. En la

\begin{tabular}{|c|c|}
\hline$C_{O E}(\mathrm{ppm})$ & $\boldsymbol{P}_{\text {Trtabajo }}$ (Bar) \\
\hline 100 & 0,070 \\
\hline 200 & 0,140 \\
\hline 300 & 0,210 \\
\hline 400 & 0,280 \\
\hline 500 & 0,350 \\
\hline 600 & 0,420 \\
\hline 700 & 0,490 \\
\hline 800 & 0,560 \\
\hline 900 & 0,630 \\
\hline
\end{tabular}
Tabla 1 se presentan las presiones de la cámara variando la concentración $\mathrm{OE}_{(\mathrm{g})}$ a $T_{\text {trabajo }}=60^{\circ} \mathrm{C}$ en

\section{Presión en función de la humedad} relativa:

Teniendo en cuenta que la mezcla de gases es un gas húmedo y la humedad relativa es la relación entre la presión presente en el aire y la cantidad máxima de presión que el aire puede mantener teóricamente a una temperatura específica, de la Ecuación 2 tenemos:

$$
\% H R_{a m b}=\frac{P_{T_{-} a m b}}{P_{S_{-} a m b}} \times 100
$$

Asumiendo $\% H R_{\text {trabajo }}$ óptimo de 60\% (Agalloco y Carleton, 2007), entonces la Ecuación 19 queda como:

$$
P_{\text {Strabajo }}=1,67 P_{\text {Ttabajo }}
$$

En la Tabla 2 se muestran las diferentes presiones de vapor de agua en función de la presión de trabajo planteada en la Ecuación 20.

TABLA 2. EFECTO DE PRESIÓN DE TRABAJO $Y$ CONCENTRACIÓN DE OE ${ }_{\left({ }^{\prime}\right)^{\prime}}$ EN LA PRESIÓN DE VAPOR DE AGUA A UNA $T_{\text {trabajo }}=60^{\circ} \mathrm{C}, \mathrm{K}=36600 \mathrm{G} / \mathrm{MOL} \mathrm{Y} R=$ 0,08314 BAR.L/MOL.K

\begin{tabular}{c|c|c|}
$\boldsymbol{C}_{O E}(\mathbf{p p m})$ & $\boldsymbol{P}_{\text {Ttrabajo }}$ (Bar) & $\boldsymbol{P}_{\text {strabajo }}$ (Bar) \\
\hline 100 & 0,070 & 0,117 \\
\hline 200 & 0,140 & 0,233 \\
\hline 300 & 0,210 & 0,350 \\
\hline 400 & 0,280 & 0,467 \\
\hline 500 & 0,350 & 0,583 \\
\hline 600 & 0,420 & 0,700 \\
\hline 700 & 0.490 & 0,817 \\
\hline 800 & 0.560 & 0,933 \\
\hline 900 & 0.630 & 1,050 \\
\hline
\end{tabular}

Tiempo letal 90 (Valor D) en función de la concentración de $\mathrm{OE}$

Para encontrar el Valor-D en función de la concentración, se debe tener en cuenta el Valor $D_{\text {Re }}$ el cual está dado por la ficha técnica de los indicadores biológicos para Bacillus atropheus, de la Ecuación 3:

$$
\text { Valor } D * C_{O E}=k
$$


Entonces para el Valor-D y Valor- $\mathrm{D}_{\text {Ref, }}$ la constante $k$ es la misma, por lo tanto:

$$
\text { Valor } D * C_{O E}=\text { Valor } D_{\text {Ref }} * C_{\text {Ref }}=
$$

Despejando la Valor-D:

$$
\text { Valor } D=\frac{\text { Valor } D_{\text {Ref. }} * C_{\text {Ref }}}{C_{O E}}
$$

En la Tabla 3 se presentan los Valor-D para $\mathrm{Ba}$ cillus atropheus a una $\% \mathrm{HR}=60 \%, \mathrm{C}_{\mathrm{Ref}}=700 \mathrm{ppm} \mathrm{y}$ Valor- $\mathrm{D}_{\text {Ref }}=2,83 \mathrm{~min}$.

TABLA 3. EFECTO DE LA CONCENTRACIÓN DE OE $E_{(q)}$ EN EL TIEMPO LETAL 90

\begin{tabular}{c|c|}
$\boldsymbol{C}_{\mathbf{O E}}(\mathbf{p p m})$ & Valor $\boldsymbol{D}(\mathbf{m i n})$ \\
\hline 100 & 19,81 \\
\hline 200 & 9,91 \\
\hline 300 & 6,60 \\
\hline 400 & 4,95 \\
\hline 500 & 3,96 \\
\hline 600 & 3,30 \\
\hline 700 & 2,83 \\
\hline
\end{tabular}

Para una $C_{O E}=600 \mathrm{ppm}$, como concentración de trabajo el Valor $D=3,30$, a partir de este valor, se recalcularon los valores de referencia $\left(\right.$ Valor $D_{\text {Ref }}$ ) en el rango de concentraciones (300-750 ppm) como se muestra en la Tabla 4.

TABLA 4. VALOR $D_{\text {Ref }}$ EN EL RANGO LINEAL DEL CAMBIO DE LA TASA DE LETALIDAD MICROBIANA

\begin{tabular}{c|c|}
$\boldsymbol{C}_{\text {Ref }}$ (ppm) & Valor $\boldsymbol{D}_{\text {Ref }}$ (min) \\
\hline 300 & 6,60 \\
\hline 350 & 5,66 \\
\hline 400 & 4,95 \\
\hline 450 & 4,40 \\
\hline 500 & 3,96 \\
\hline 550 & 3,60 \\
\hline 600 & 3,30 \\
\hline 650 & 3,05 \\
\hline 700 & 2,83 \\
\hline 750 & 2,64 \\
\hline
\end{tabular}

Tiempo letal 90 (Valor D) en función de la temperatura

Despejando el Valor D de la Ecuación 5 se tiene:

$$
\text { Valor } D=\text { Valor } D_{\text {Ref }} * 10\left(\frac{54^{\circ} \mathrm{C}-T_{\text {trabajo }}}{31^{\circ} \mathrm{C}}\right)
$$

En la Tabla 5 se presentan los Valores D encontrados con la Ecuación 24, estos valores son muy cercanos a los reportados por Agalloco \& Carleton (2007) para las concentraciones de referencia a

\begin{tabular}{|c|c|c|c|c|}
\hline $\begin{array}{c}C_{\text {Ref }} \\
(\mathbf{p p m})\end{array}$ & $\begin{array}{c}\text { Valor } D_{\text {Ref }} \\
\text { (min) }\end{array}$ & $\begin{array}{c}T_{\text {trabajo }} \\
\left({ }^{\circ} \mathrm{C}\right)\end{array}$ & $\begin{array}{c}\text { Valor D } \\
(\mathrm{min})\end{array}$ & $\begin{array}{c}\text { Valor D (min) } \\
\text { Agalloco \& } \\
\text { Carleton, } 2007\end{array}$ \\
\hline \multirow{3}{*}{300} & \multirow{3}{*}{6,60} & 40 & 18,67 & 18,11 \\
\hline & & 54 & 6,60 & 6,37 \\
\hline & & 60 & 4,23 & 4,44 \\
\hline \multirow{3}{*}{450} & \multirow{3}{*}{4,40} & 40 & 12,45 & - \\
\hline & & 54 & 4,40 & 4,30 \\
\hline & & 60 & 2,82 & - \\
\hline \multirow{3}{*}{750} & \multirow{3}{*}{2,64} & 40 & 7,47 & 8,33 \\
\hline & & 54 & 2,64 & 2,84 \\
\hline & & 60 & 1,69 & 1,92 \\
\hline
\end{tabular}
300,450 y 750 ppm, y temperaturas de trabajo 40, 54, $60 \stackrel{\circ}{\circ} \mathrm{C}$ sobre Bacillus arthophaeus ATTC \# 9372.

\section{Tasa de letalidad}

Dado que la Ecuación 6 no tiene en cuenta la humedad relativa, se modeló la tasa de letalidad en función del \%HR, a diferentes temperaturas como se muestra en la Figura 1.

En la Figura 1 se puede observar que la tasa de letalidad en función del \%HR, independiente de la temperatura describe una función sigmoide que se pudo representar con la siguiente ecuación:

$$
T L=T L_{R e f} \frac{\mathrm{e}^{\left(-2\left(\% H R_{a m b}-60 \%\right)\right)}}{\left(1+\mathrm{e}^{\left(-2\left(\% H R_{a m b}-60 \%\right)\right)}\right)^{2}}
$$




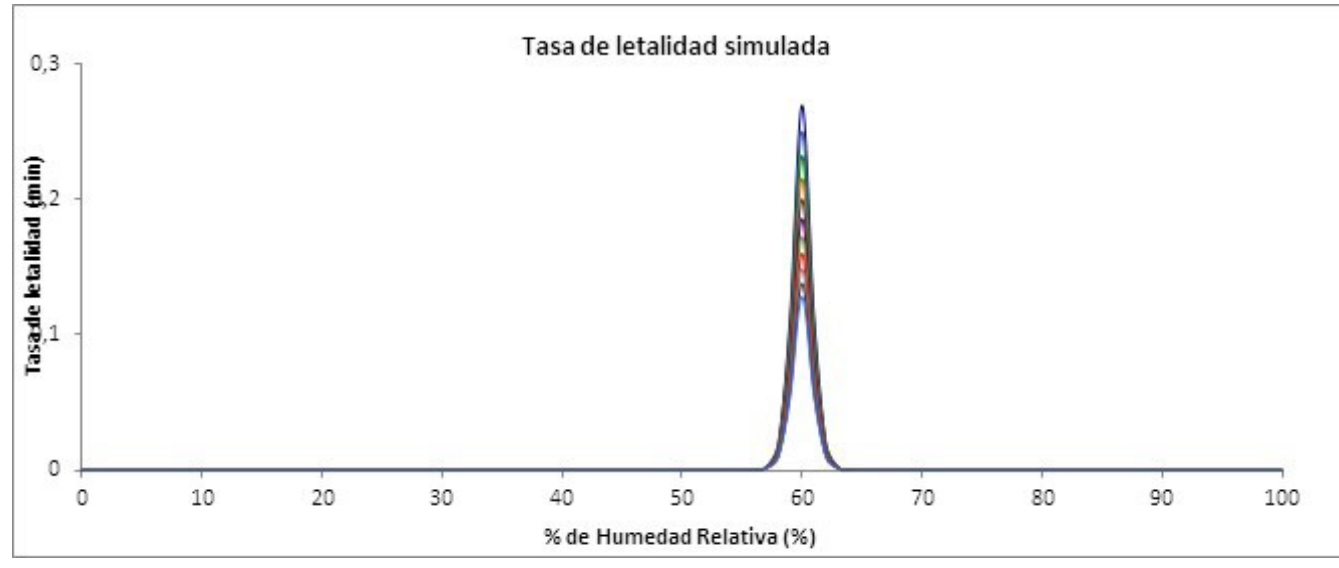

La máxima tasa de letalidad (mayor muerte de microorganismos) se obtuvo a una $\% H R=60 \% \mathrm{y}$ una tasa de cambio de letalidad de 2 , lo que quiere decir que a un incremento de $10{ }^{\circ} \mathrm{C}$ se duplica la velocidad la reacción (Agalloco y Carleton, 2007). De esta forma se ha demostrado que un $\% H R=60 \%$ se puede tomar como $\% H R_{R e f}$ para la esterilización con $\mathrm{OE}$, permitiendo incluir la humedad en el modelo que describe el comportamiento de la muerte de los microorganismos.

\section{Inactivación microbiana}

El proceso de inactivación microbiana describe un comportamiento cinético de primer orden, para un numero de esporas de Bacillus arthophaeus (Russel, Hugo, y Avliffe, 2004; Agalloco y Carleton, 2007), como se ilustra en la Ecuación 26 :

$$
\operatorname{Ln}\left(N_{t}\right)=\operatorname{Ln}\left(N_{o}\right)-(\text { Valor } K) \cdot t
$$

Donde $N_{t}$ es el número de esporas en el tiempo $t, N_{o}$ es el número inicial de esporas de Bacillus atropheus y el Valor K es la constante de inactivación se expresa como:

$$
\text { Valor } K=\frac{2,303}{\text { Valor } D}
$$

Remplazando el Valor D de la Ecuación 24 en la Ecuación 27 tenemos:

$$
\text { Valor } K=\frac{2,303}{\text { Valor } D_{\text {Ref }} * 10\left(\frac{54^{\circ} \mathrm{C}-T_{\text {trabajo }}}{31^{\circ} \mathrm{C}}\right)}
$$

Modificando la Ecuación 26 se tiene:

$$
N_{t}=N_{0} \mathrm{e}^{- \text {Valor } K * t}
$$

Reemplazando la Ecuación 28 en la Ecuación 29:

$$
N_{t}=N_{0} \mathrm{e}\left(\frac{2,303}{\text { Valor } D_{\text {Ref }} * 10\left(\frac{54^{\circ} \mathrm{C}-T_{\text {trabajo }}}{31^{\circ} \mathrm{C}}\right)}\right) * t .
$$

En la Figura 2 se muestran las isotermas de letalidad para el número de esporas de Bacillus arthophaeus de $3,5 \times 10^{6}$, para un Valor $D_{\text {Ref }}=6,6$. El modelo planteado en la Ecuación $\mathbf{3 0}$ a diferentes temperaturas en un ciclo de esterilización sigue una distribución exponencial.

Teniendo en cuenta la tasa de letalidad encontrada en función de la \%HR, de la Ecuación 25, y con el fin de conservar el comportamiento cinético de primer orden de la Ecuación 26, incorporando la relación entre la tasa de letalidad y la tasa de letalidad de referencia en el segundo término tenemos:

$$
\operatorname{Ln}\left(N_{t}\right)=\operatorname{Ln}\left(N_{0}\right)-(\text { Valor } K) * t * \frac{T L}{T L_{R e f}}
$$


Figura 2. Isotermas de letalidad para el número de esporas de Bacillus arthophaeus en función del tiempo

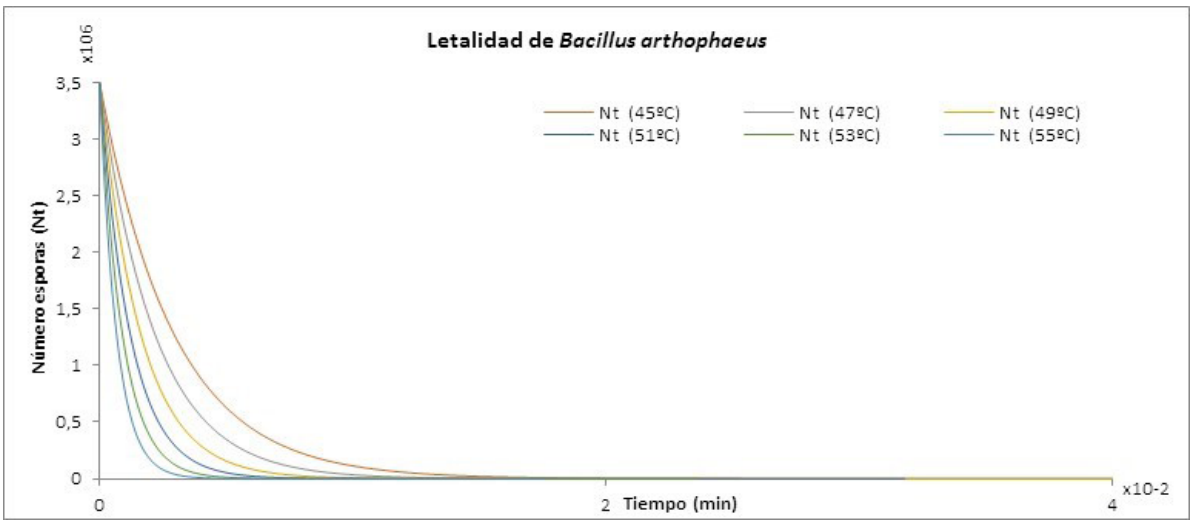

Reemplazando las Ecuaciones 28 y 29 en la 31:

$$
\operatorname{Ln}\left(N_{t}\right)=\operatorname{Ln}\left(N_{0}\right)-\frac{2,303}{{\text { Valor } D_{\text {Ref }} * 10}^{*}\left(\frac{54^{\circ} \mathrm{C}-T_{\text {trabajo }}}{31^{\circ} \mathrm{C}}\right)} \cdot t \cdot \frac{\mathrm{e}^{\left(-2\left(\% H R_{a m b}-60 \%\right)\right)}}{\left(1+\mathrm{e}^{\left(-2\left(\% H R_{a m b}-60 \%\right)\right)}\right)^{2}}
$$

De la Ecuación 32 se tiene que el número de $\quad \mathrm{y} \% H R_{a m b}$ como se muestra en la Ecuación 33:

microorganismos en el tiempo, depende de la $T_{\text {trabajo }}$

$$
N_{t}=N_{0} \mathrm{e}\left(\frac{2,303}{\text { Valor } D_{\text {Ref }}^{*}{ }^{*} 10\left(\frac{54^{\circ} \mathrm{C}-T_{\text {trabajo }}}{31^{\circ} \mathrm{C}}\right)}\right)\left(\frac{\mathrm{e}^{\left(-2\left(\% H R_{a m b}-60 \%\right)\right)}}{\left(1+\mathrm{e}^{\left(-2\left(\% H R_{a m b}-60 \%\right)\right)}\right)^{2}}\right)
$$

Modelación de la letalidad en la cámara de esterilización

Con el programa MATLAB se modeló la Ecuación 33, construyendo una interfaz gráfica amigable con el fin de calcular el tiempo en los ciclos para la esterilización con óxido de etileno.
En las Figuras 3 y 4 se muestran la interfaz gráfica del efecto de la temperatura de trabajo en el número de esporas de Bacillus arthophaeus, teniendo como parámetros óptimos tales como: $N_{0}=$ $3,5 \times 10^{6}, C_{R e f}=700 \mathrm{ppm}, T_{a m b}=23{ }^{\circ} \mathrm{C}, \% H R=60 \%$ y $P_{\text {trabajo }}=0,490 \mathrm{~atm}$, Valor $\mathrm{D}=3,3 \mathrm{~min}\left(\right.$ Valor $D_{\text {Ref }}=$ 6,6 $\mathrm{min})$.

\section{Figura 3. Interfaz gráfica en MATLAB para la determinación del ciclo de esterilización con OE a $T_{\text {trabajo }}=55^{\circ} \mathrm{C}$}

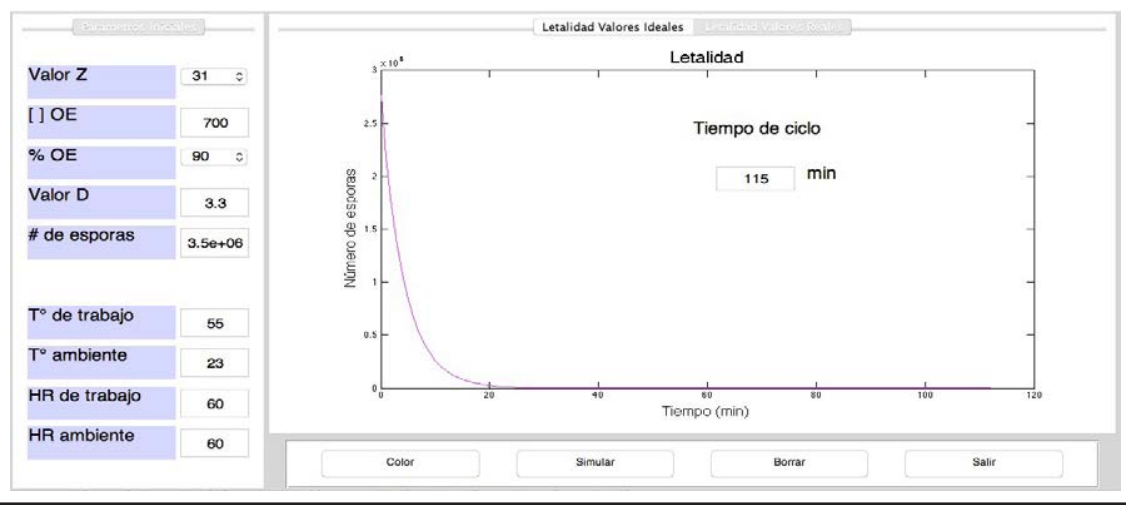




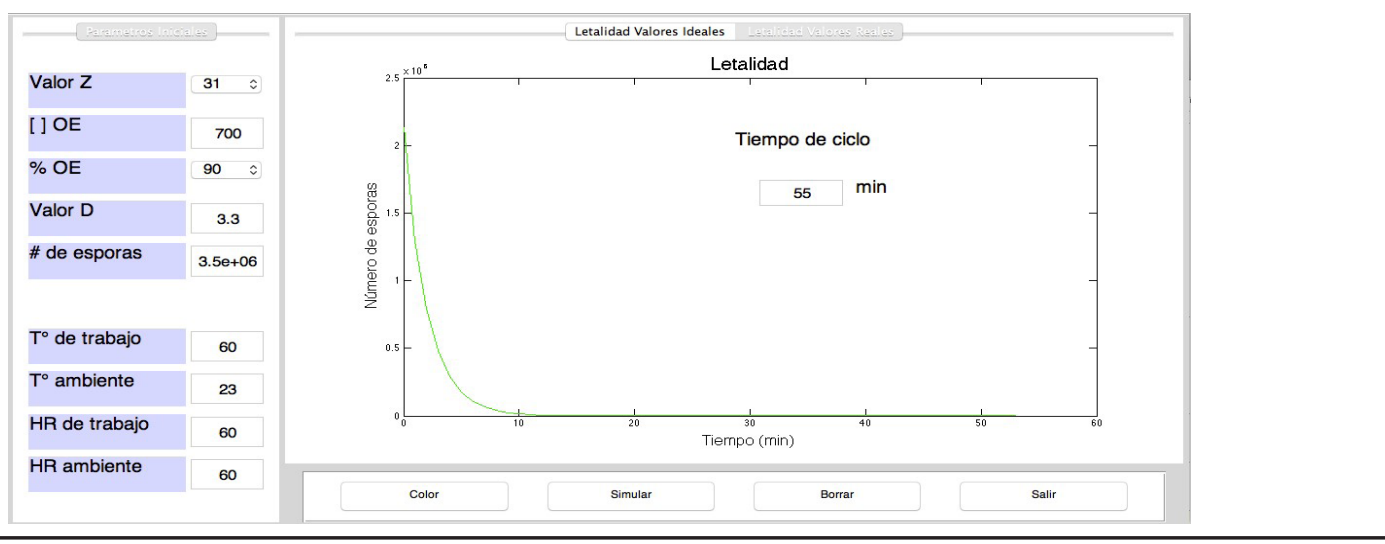

Para la $T_{\text {trabajo }}=55^{\circ} \mathrm{C}$ se presentó un ciclo de 115 minutos (Figura 3) comparado con la $T_{\text {trabajo }}=$ $60{ }^{\circ} \mathrm{C}$, donde el ciclo fue de 55 minutos (Figura 4). Por lo tanto se tiene un optimización del ciclo en 60 minutos, recomendando una $T_{\text {trabajo }}=60^{\circ} \mathrm{C}$, para la aplicación del proceso de esterilización.

\section{CONCLUSIONES}

Con el algoritmo desarrollado en MATLAB, se determinaron las condiciones como: la concentración de óxido de etileno $\left(C_{R e f}=700 \mathrm{ppm}\right)$, humedad relativa $\left(\% H R_{\text {Ref }}=60 \%\right)$ y temperatura de trabajo $\left(T_{\text {trabajo }}=23{ }^{\circ} \mathrm{C}\right)$, seguido del ciclo óptimo (55 minutos). A demás el algoritmo permitió realizar cálculos haciendo uso del Valor D en función de la temperatura y humedad, indicando que son factores importantes a tener en cuenta en la tasa de letalidad $\mathrm{Ba}$ cillus arthophaeus. El modelo puede ser de utilidad, dado que proporciona a los interesados la capacidad de optimizar un proceso de esterilización, evitando el uso excesivo de suministros como el óxido de etileno y el tiempo del ciclo, con el fin de asegurar una máxima letalidad de los microorganismos en dispositivos médicos.

\section{AGRADECIMIENTOS}

Los autores expresan su agradecimiento a la Convocatoria 645 "Convocatoria Nacional Jóvenes Investigadores e Innovadores año 2014” de Colciencias, por la financiación del proyecto en el marco del convenio especial de cooperación número 0215 de 2014.

\section{REFERENCIAS}

Acosta-Gnass, S., Stempliuk, V., (2008). Manual de esterilización para centros de salud, Organización Panamericana de la Salud.

Agalloco, J., Carleton, F.J., (2008). Validation of Pharmaceutical Processess. doi:10.1081/E-EPT-100001927

Forero, M. teresa, (1997). CONDUCTAS BASICAS EN BIOSEGURIDAD: MANEJO INTEGRAL. Protocolo Básico para el Equipo de Salud. MInisterio de Salud 1-51.

Russel, A.D., Hugo, W.B., Avliffe, G.A.J., (1999). Principles and practice of disinfection, preservation and sterilization. 3rd ed. Blackwell Science, Oxford. doi: 10.1002/9781118425831

International Standards Office, (2007) ISO 11135-1. Esterilización de productos para el cuidado de la salud. Óxido de etileno. Geneva: ISO.

Heider, D., Gomann, J., Junghann, B., Kaiser, U., (2002). Kill kinetics study of Bacillus subtilis spores in ethylene oxide sterilisation processes. Zentr Steril.

Mendes, G.C., Brandão, T.R.S., Silva, C.L.M., (2012). Ethylene oxide (EO) sterilization of healthcare products, in: Sterilisation of Biomaterials and Medical Devices. Elsevier Ltd., pp. 71-96. doi:10.1016/B978-184569-932-1.50004-0

Mendes, G.C.C., Brandão, T.R.S., Silva, C.L.M., (2007). Ethylene oxide sterilization of medical devices: A review. American Journal of Infection Control. doi:10.1016/j.ajic.2006.10.014 
Rutala, W.A., Gergen, M.F., Weber, D.J., (1998). Comparative evaluation of the sporicidal activity of new low- temperature sterilization technologies: Ethylene oxide, 2 plasma sterilization systems, and liquid peracetic acid. American Journal of Infection Control 26, 393398. doi:10.1016/S0196-6553(98)70034-3

\section{PARA CITAR ESTE ARTÍCULO TO REFERENCE THIS ARTICLE / PARA CITAR ESTE ARTIGO /}

Jaramillo-Ruiz, N.; Araque-Marín, P.; Montoya-Góez, Y. (2017). Modelo matemático de letalidad sobre Bacillus arthophaeus en procesos de esterilización de dispositivos médicos con óxido de etileno. Revista EIA, 14(28), julio-diciembre, pp. 4555. [Online]. Disponible en: https://doi.org/10.24050/reia. v14i28.974 